\title{
BOOK REVIEW: \\ QUESTIONNAIRES IN SECOND LANGUAGE RESEARCH
}

\author{
Rahmadi Nirwanto \\ STAIN Palangka Raya \\ rnirwanto@yahoo.com
}

\begin{tabular}{l} 
Questionnaires in Second Language \\
Research: Construction, Administration, \\
and Processing ( ${ }^{\text {nd }}$ ed.) \\
\hline Zoltan Dornyei \&Tatsuya Taguchi \\
\hline Routledge 2010, xiii + 183 pp. \\
\hline ISBN 0-203-86473-5
\end{tabular}

In the world of research specifically in second language or applied linguistics, questionnaires are widely used and the most often employed for data collection devices. The quality of questionnaires will affect the quality of the data needed for answering research problems. If the questionnaires are well-designed, the data will be valid and accurate. On the other hand, if the questionnaires are poorly-designed, the data will be invalid and inaccurate. In order to construct well-developed questionnaires, the questionnaire constructors must follow certain theoretical guidelines or practical principles. These can be found in the book entitled, "Questionnaires in Second Language Research: Construction, Administration, and Processing." By reading the book, the readers will have in-depth understanding of the theoretical and practical principles of constructing questionnaires. I will review the content of the chapters and highlight some important issues related to the content of the books.

The book is written by Zoltan Dornyei, a professor of Psycholinguistics in the School of English Studies at the University of Nottingham, UK. He found that questionnaire theory is very helpful in his research. Another writer is Tatsuya Taguchi, a Ph.D Candidate in the School of English Studies at the University of
Nottinghm, UK. Tatsuya has helped him to update the references and improve several other parts of the material particularly the addition of a section on translating questionnaire items in Chapter 2 and the discussion of how to collect survey data on the Internet in Chapter 3.

The current book is the new revision (the second edition) of the previous book. In this edition, the writers make some significant changes including adding a whole new chapter to the book, Chapter 5 talking about "Illustration: Developing a Motivation Questionnaire," to provide a detailed and illustrative analysis of how an actual scientific research is developed from scratching following theoretical guidelines.

According to Zoltan Dornyei and Tatsuya Taguchi, the book offers an overview of theory of questionnaire design, administration, and processing, and it is accessibility made by concrete and real-life second language research applications. The second edition features a new chapter on how an actual scientific instrument was developed using theoretical guidelines in the book, and provides new sections on translating questionnaires and collecting survey data on the Internet. The book is useful for both researchers and students in second language studies, applied linguistic, and TESOL programs to construct and apply questionnaires as a research instrument.

Structurally, the book consists of 5 (five) chapters. Each chapter has its own sections. Chapter 1 discusses questionnaires in Second Language Research. Then it is followed by several sections: (1.1) What Are "Questionnaire" and What Do They Measure? and (1.2) Using 
Questionnaire Pro and Cons Questionnaires in Quantitative and Qualitative Research.

In Section 1.1, the readers are offered the concept of questionnaires. It is stated that "Questionnaires are any written instruments that present respondents with a series of questions or statements to which they are to react either by writing out their answers or selecting from among existing answers " (Brown in Dornyei, 2010). These concepts enable the readers to be able distinguish between questionnaires belong to technical terms in Second Language or Applied Linguistics studies and questionnaires belong to common sense. In this book, the writers compare between a test and questionnaires. A test measures how well someone can do something. In contrast, questionnaire does not have good or bad answers. In addition, questionnaires are often referred names such as "inventories," "forms," "opinionnaires," "tests," "batteries," "checklists," "scales," "surveys," "schedules," ... "studies," "profiles," "indexes/indicators," or even simply "sheets." In second language research, Dornyei agrees with Brown who defines questionnaires as research instruments for measurement purposes to collect reliable and valid data. This sub-chapter is then followed by the discussions of "What questionnaires Measure," and "Using Questionnaire Pro and Cons Questionnaires in Quantitative and Qualitative Research."

Related to the topic in Section 1.1., "What questionnaires measure provides the areas to be covered by questionnaires," Dornyei (2010:5-6) points out those questionnaires can yield three types of data about the respondent: (1) Factual, behavioral, and attitudinal. Factual questions (also called "classification" questions or "subject descriptors") are used to find out about who the respondents are. They typically cover demographic characteristics (e.g., age, gender, and race), residential location, marital and socioeconomic status, level of education, religion, occupation as well as any other background information that may be relevant to interpreting the findings of the survey. (2)
Behavioral questions are used to find out what the respondents are doing or have done in the past. They typically ask about people's actions, lifestyles, habits, and personal history. Perhaps the most well-known questions of this type in L2 studies are the items in language learning strategy inventories that ask about the frequency of the use of a particular strategy in the past. (3) Attitudinal questions are used to find out what people think. This is a broad category that concerns attitudes, opinions, beliefs, interests, and values. These five interrelated terms are not always distinguished or defined very clearly in the literature. The term "Attitudes" concerns evaluative responses to a particular target (e.g., people, institution, and situation). The term "Opinions" is just as subjective as attitudes, but they are perceived as being more factually based and more changeable. People are always aware of their opinions but they may not be fully conscious of their attitudes. The term "Beliefs" has a stronger factual support than opinions and often concerns the question as to whether something is true, false, or right. The term "Interests" is preference for particular activities. The term "Values" on the one hand, concerns preferences for "life goals" and "ways of life." In order to be able to understand these terms, find more in the book.

If the readers are interested in more about the technical aspects of the questionnaires, they can read Chapter 2. This is a crucial chapter because it deals with constructing questionnaires. The chapter 2 is divided into 10 (ten) sections which start with the discussion of Section (2.1) containing "General Feature." According to Dornyei, we need to take a number of important decisions regarding the general features of the would-be instrument. First of all, we need to specify the maximum length of time that the completion of the questionnaire could take, then we need to consider general format characteristics, and finally we need to think about the issue of anonymity particularly if we are going to target sensitive/ confidential topics. Then "The Main Parts of a Questionnaire" is discussed 
in the Section (2.1). In his classification, there are several parts of a questionnaire including "Title", “Instructions", "Questionnaire Items", "Additional Information", and a Final "Thank You". The discussions of title enable the readers to identify the domain of the investigation, to provide the respondent with initial orientation, and to activate various content schemata. In terms of instructions, the readers are guided to write instructions because they can play an important role in determining how respondents go about answering the items. After studying about the instructions, the readers can come to the central part of the questionnaire, the actual items. At the end of the questionnaires, the readers are guided how to give additional information. Finally, the readers are reminded to thank the respondents for their participations.

Constructing a good questionnaire requires a series of steps or procedures. The chapter presents a number of steps or procedures. They are included in the discussions of "Appropriate Sampling of the Questionnaire Content" and "the Significance of Multi-Item Scales" (in Section 2.3), "Closed-Ended Questionnaire Items" (in Section 2.4), and "Open-Ended Questionnaires" (in Section 2.5). In a more detail, Section 2.4 comprises of rating scales (2.4.1), multiple choices items (2.4.2), rank order items (2.4.3), numeric items (2.4.4), and check lists (2.4.5). Section 2.5 includes open questions (2.5.1), clarification question (2.5.2), sentence completion items (2.5.3), and short-answer question (2.5.4).

Writing good items is a key success for researchers to obtain information from the respondents. Section 2.6 emphasizes on "How to Write Good Items". In this section, the discussions of "Drawing Up an Item Pool", "Rules about Writing Item", and "Sensitive Items" are raised in the Section 2.6.2, and 2.6.3. In terms of the item pool, Dornyei points out that those successful item designers heavily rely on their own verbal creativity, but they also draw on two additional sources: (1) Qualitative, exploratory data gathered from respondents, such as notes taken during talks and brainstorming in focus or discussion groups; recorded unstructured/ semi-structured interviews; and student essays written around the subject of the enquiry (see e.g., Tseng et al., 2006), and (2) borrowing questions from established questionnaires. Questions that have been frequently used in advance must have been through extensive piloting and therefore the chances are that "most of the bugs will have been ironed out of them"

After reading Section 2.6, the readers can read Section 2.7 which discusses ordering principles. The discussion includes the Section 2.7.1 "Clear and Orderly Structure". If the ordering of questions is unpredictable or seemingly haphazard, it will frustrate respondents. The Section 2.7.2 discusses "Opening Question". It needs to be interesting, somewhat simple, focuses on a relatively mild or neutral aspect and certainly not threatening or sensitive.

The topic on translating the questionnaire is discussed in the Section 2.8. Dornyei (2010:48) mentions somereasons, among others are (1) many of the available established L2 questionnaires are published in English; (2) cross-cultural studies often require the administration of the same instrument to samples of different ethnolinguistic origin; and (3) there are many multi-national research teams (including supervisor-research student teams) that prepare the original version of a questionnaire in one language (often English) that it is translated into the language(s) of the participants before. Section 2.8 is divided into two parts: (2.8.1) Translation as a Team-Based Approach and (2.8.2) Translation with Limited Resources.

Some software companies have developed computer program to cater the need of various institutions which utilize questionnaire for different purposes, Section (2.9) focuses on "Computer Programs for Constructing Questionnaire". In the final section of Chapter 2, it discusses the topic, "Piloting the Questionnaire and Conducting Item Analysis" (in Section 2.10). Then the Section 2.10 contains the discussion of "Initial Piloting of the Item Pool" (in Section 
2.10.1), "Final Piloting" (in Section 2.10.2), and "Item Analysis" (in Section 2.10.3).

According to the writers, the most frequent questions asked by novice researchers who have a planning to use questionnaires in their investigation are: first, "How many people do I need to survey?" In measurement terms this question can be formulated as $\mathrm{Y}$ "How large should my sample be?" And second, "What sort of people shall I select?" Or, in other words, "Who shall my sample consist of?" The answers to these questions can be found in the section (3.1.1) Sampling procedures, and (3.1.2) the size of sample and specific discussion on potential pitfalls that might put the validity of participant self-selection. The different of questionnaire administrations are in the Section (3.2.1) administration by mail, (3.2.2) one to one administration, and (3.2.4) one line administration.

Chapter 3 deals with the topics: (3.1) Selecting the sample, (3.2) Main Types of Questionnaire Administration, (3.3) Strategies to Increase the Quality, and Quantity of Participants Response. This section contains the following topics: (3.3.1) Advanced Novice, (3.3.2) Attitudes, Conveyed by Teachers, Parents, and Other Authority Figures, (3.3.3) Respectable Sponsorship, (3.3.4) The Presence of a Survey Administrator, (3.3.5) The Behavior of a Survey, (3.3.6) Communicating the Purpose and significance of the Survey, (3.3.7) Emphasizing Confidentiality, (3.3.8) Reading Out the Questionnaire Instructions, (3.3.9)The Style and Layout of the Questionnaire, (3.3.10) Promising Feedback on the Results. The Section 3.4 contains: (3.4.1) Basic Ethical Principles of Data Collection, (3.4.2) Obtaining Consent for Children, and (3.4.3) Strategies for Getting around Anonymity.

TheSection 4.1 discusses Coding Questionnaire Data includes (4.1.1) First Things First: Assigning Identification Codes, and (4.1.2) Coding Quantitative Data. The Section 4.2 covers (4.2.1) Creating and Naming the Data File and (4.2.2) Keying in the Data. This Section consists of (4.3.1) Data Cleaning and (4.3.2) Data
Manipulation, (4.3.3) Reducing the Number of Variables in the Questionnaire, (4.3.4) Main Types of Questionnaire Data, (4.3.5) Examining the Reliability and Validity of the Data, and (4.3.6) Statistical Procedures to Analyze Data. The Section 4.6 includes (4.6.1) General Guidelines, (4.6.2) Technical Information to Accompany Survey Results, and (4.6.3) Reader-Friendly Data Presentation Methods. The Section 4.7 contains (4.7.1) Questionnaire Survey with Follow-up Interview Retrospection and (4.7.2) Questionnaire Survey Facilitated by Preceding Interview. The Section 5.1 contains (5.1.1) Deciding the Content Areas to be Covered in the Questionnaire, (5.1.2) Designing Items for the Item Pool, (5.1.3) Designing Rating Scales, (5.1.4) Designing the Personal Information, (5.1.5) Designing Instructions, (5.1.6) Designing the Questionnaire Format, and (5.1.7) Grouping and Organizing Items and Questions. The Section 5.3 contains (5.3.1) Missing Values and the Range of Responses, (5.3.2) The Internal Consistency Reliability of the Initial Scales, and (5.3.3) Modification of the Personal Information Items.

Different from the previous chapters (Chapter 1,2 , and 3 which are concerned with theoretical principles), the Chapter 4 focuses on the procedures covering the following topics: (4.1) Coding Questionnaire Data, (4.2) Inputting the Data, (4.3) Processing Closed Questionnaire, (4.4) Content Analysis of Open-Ended Questions, (4.5) Computer Program for Processing Questionnaire Data, (4.6) Summarizing and Reporting Questionnaire Data, and (4.7) Complementing Questionnaire Data with Other Information. The Chapter 5 contains Illustration: Developing a Motivation Questionnaire consists of (5.1) Construction the Initial Questionnaire, (5.2) Translating and Initial Piloting, (5.3) Final Piloting and Item Analysis, (5.4) The Final Version of the Japanese Questionnaire and Post Hoc Item Analysis, and (5.5) Adapting the Questionnaire for Use in China and Iran.

In conclusion section, the writers emphasize that the book is intended to serve practical 
purposes for the readers and draw up a check list of what they consider the most important points and recommendations for every phase of the questionnaire survey. The check list covers constructing, administering, and processing questionnaire data. 